\title{
Kongressbericht - Congress Report
}

Gemcitabine - ein Antimetabolit mit einer neuen Kombination von Wirksamkeit und Verträglichkeit

Gemcitabine, ein Antimetabolit mit einer neuen Kombination von Wirksamkeit und Verträglichkeit, war kürzlich das Thema eines Begleitsymposiums anläßlich der gemeinsamen Jahres-tagung der Deutschen und Österreichischen Gesellschaft für Hämatologie und Onkologie in Wien. Die Substanz mit der chemischen Bezeichnung 2',2'-Difluordeoxycytidin hat in einer Reihe von klinischen Phase-II-Studien bereits in der Mono-therapie gute Wirksamkeit bei soliden Tumoren gezeigt. Im Vordergrund stehen die Indikationen nichtkleinzelliges Bronchialkarzinom, metastasiertes Mammakarzinom und Ovarial-karzinom.

Die Remissionsrate beim metastasierten Mammakarzinom liegt mit 25-32\% in einer Größenordnung, die vergleichbar ist mit der eines konventionell dosierten Anthrazyklins. Beim fortgeschrittenen nichtkleinzelligen Bronchialkarzinom wurde mit einer Response-Rate von 22\% der obere Bereich dessen erreicht, was in der Monotherapie bei dieser Indikation mög-lich ist, berichtete Ulrich Gatzemeier, Großhansdorf. In einer dänischen Studie lag die Remissionsrate beim Ovarialkar-zinom bei 20\%. Hervorzuheben ist, daß dort 7 von 8 Patien-tinnen mit einem platinrefraktären Tumor auf die Gemcita-bine-Therapie ansprachen. Bei der Bewertung der Ergebnisse ist zu beachten, daß in alien Gemcitabine-Studien die Response-Daten von einem externen, internationalen Review-Board begutachtet wurden.

Gemeinsames Merkmal aller Studien ist außerdem das gün-stige Verträglichkeitsprofil der Substanz, und zwar sowohl im hämatologischen als auch im nichthämatologischen Bereich. So waren insbesondere beim metastasierten Mammakarzinom und beim nichtkleinzelligen Bronchialkarzinom Thrombo-zytenreduktionen gering, Übelkeit und Erbrechen relativ gering ausgeprägt, und mehr als 80\% der Behandelten hatten keinerlei Haarausfall.

Das günstige Verträglichkeitsprofil von Gemcitabine steht vermutlich in engem Zusammenhang mit dem Wirkmecha-

nismus der Substanz, der 2 Besonderheiten aufweist: den maskierten Kettenabbruch und die selbstpotenzierende Wirkungsverstärkung. Gemcitabine wird in der Zelle zum Triphosphat umgesetzt und in die DNA eingebaut. Im Gegen-satz zu anderen Nukleosidanaloga kommt es dann aber nicht direkt zum Kettenabbruch, sondern erst nach dem Einbau eines weiteren natürlichen Bausteins. Das heißt, der zytosta-tische Wirkstoff wird an dieser Stelle maskiert und kann von Korrekturenzymen nur schwer erkannt und durch «Heraus-schneiden» unwirksam gemacht werden. Zusätzlich übt Gemcitabine einen direkten und indirekten Einfluß auf mehrere Enzyme des Zellstoffwechsels aus. Die Folge davon ist, daß -im Sinne einer echten Selbstpotenzierung - die Konzentration des natürlichen Konkurrenten Deoxycytidin-Triphosphat sinkt, gleichzeitig Gemcitabine-Triphosphat vermehrt gebildet und seine Elimination verzögert wird.

Den Wirkmechanismus von Gemcitabine stellte Ralf Grune-wald, Berlin, vor. Er hat im Labor von William Plunkett im M.D. Anderson Cancer Center in Houston, Texas, über den neuen Wirkstoff geforscht. Er berichtete auch über Unter-suchungen die Verabreichungsweise der 
Substanz betreffend. Derzeit wird Gemcitabine in alien klinischen Studien als 30minütige Kurzzeitinfusion einmal wöchentlich verabreicht; dies 3 Wochen lang, gefolgt von einer Woche Pause, entspricht einem Zyklus. Die Dosierung lag in den Monotherapiestudien beim metastasierten Mammakarzinom und beim Ovarialkarzi-nom bei 800 mg/m2, beim nichtkleinzelligen Bronchialkarzinom bei 1250 mg/m2. Die Tatsache, daß die geplante Dosis bei den meisten Patienten eingehalten, bei einigen sogar noch gesteigert werden konnte und gleichzeitig die Therapie gut vertragen wurde, läßt weitere Optionen für Gemcitabine of-fen. Diese könnten sein: Dosiseskalation, gegebenenfalls mit verändertem Verabreichungsschema, sowie Kombination mit anderen Zytostatika oder einer Strahlentherapie. Der beson-dere Wirkmechanismus von Gemcitabine läßt für - derzeit bereits weltweit laufende Kombinationsstudien einen syner-gistischen Effekt erwarten.

K. Possinger, Berlin

(C) 1995 S. Karger GmbH, Freiburg 